\title{
Modelling phenotypic plasticity. II. Do genetic correlations matter?
}

\author{
MASSIMO PIGLIUCCI \\ Departments of Botany and of Ecology \& Evolutionary Biology, University of Tennessee, Knoxville, TN 37996-1100, \\ U.S.A.
}

\begin{abstract}
Predictions of the evolutionary trajectory of reaction norms and interpretation of empirical results are usually based on two mathematically equivalent ways of partitioning phenotypic variance into its genetic, environmental, and interaction components: the genotype by environment interaction estimated by means of an analysis of variance, or the interenvironment genetic correlation (i.e. the genetic correlation between the expressions of the same trait in two environments). Both these quantities are supposed to indicate the amount of genetic variability for plasticity in a natural population. I point out that not only are the qualitative predictions based on these statistical methods sometimes in conflict with each other, but that both may fail to predict rates of evolution and equilibria under some circumstances, because they ignore the details of the genetic machinery. It is shown that, ultimately, the only way to predict reliably the evolution of plasticity is actually to know its specific genetic basis and the genotypic constitution of the population, however inconvenient this may be from both theoretical and empirical standpoints. The discussion is framed in terms of a simple one-locus two-allele model that mimics the real case of the pennant/vestigial system describing plasticity of wing length to temperature in Drosophila melanogaster.
\end{abstract}

Keywords: genetic correlations, genotype-environment interactions, phenotypic plasticity, population genetics, quantitative genetics.

\section{Introduction}

Phenotypic plasticity is the ability of a genotype to produce different phenotypes in different environments and it is a property of the norm of reaction of that genotype (Woltereck, 1909; Schmalhausen, 1949). Although very important for breeding experiments since the beginning of the century (Falconer, 1990), in recent years phenotypic plasticity has increasingly been the focus of theoretical and empirical research in evolutionary biology (reviews in Bradshaw, 1965; Schlichting, 1986; Sultan, 1987; West-Eberhard, 1989; Scheiner, 1993).

Theoretical models of the evolution of phenotypic plasticity have been proposed mostly from within the framework of quantitative genetics, i.e. they are statistical accounts of changes in mean phenotype caused by allelic substitutions at many loci, each with small and additive effects (e.g. Via \& Lande, 1985; Gillespie \& Turelli, 1989; de Jong, 1990; Gomulkiewicz \& Kirkpatrick, 1992; Gavrilets \& Scheiner, 1993). Alternative approaches have made use of optimization theory, but these are genetically equivalent to quantitative genetic models (Charlesworth, 1990; Abrams et al., 1993). All empirical studies of phenotypic plasticity quantify the genetic variation for plastic responses in one of two ways. (1) The first method uses analyses of variance, according to which the total phenotypic variance can be accounted for by three major sources of variation: genotype, environment, and genotype by environment interaction (e.g. Falconer, 1990). (2) The second method utilizes the concept of interenvironment genetic correlation, according to which a very positive or very negative correlation between the expression of the same traits in two environments implies strong constraints on the evolution of plasticity, whereas a weak or near-zero correlation corresponds to the greatest evolutionary degrees of freedom for the system (the concept was introduced by Falconer, 1952 and elaborated by Yamada, 1962; it has been used in an evolutionary context by Via \& Lande, 1985 and has been generalized to the multivariate case by van Tienderen \& Koelewijn, 1994 and de Jong, 1995). Both approaches ignore the details of the genetic machinery underlying plastic 
responses. In the case of the analysis of variance, the genetics is simply considered as a 'black box': the goal is to achieve a statistical representation of the population of interest, not to infer causal mechanisms. Evolutionary quantitative genetics sets a more ambitious agenda: not only are we interested in describing the current genetic make-up of the population, but we want to infer how such make-up is likely to constrain future evolution of the population. Several empirical papers using either method draw conclusions about the amount of genetic variation for plasticity, and the possibility of evolutionary changes given the current genetic variance-covariance structure of the population(s) (e.g. Conner \& Via, 1993; Andersson \& Shaw, 1994), although others find contradictions or difficulties in reconciling the two approaches (e.g. Ebert et al., 1993; Gebhardt \& Stearns, 1993; Windig, 1994).

A fundamental question that we need to ask when evaluating empirical data on phenotypic plasticity based on statistical models is: to what extent can we ignore or simplify the details of the mechanistic basis of phenotypes, and how does this affect our ability to interpret the past evolutionary history and predict the future one (Pigliucci, 1992)? In the following I will present a series of simple scenariograms using which I will show how little we can infer about the evolution of phenotypic plasticity by means of statistical analyses (Lewontin, 1974) especially when major loci underlie such plasticity. I will therefore call both for more cautious interpretations of analyses of variance and genetic correlations in the absence of any knowledge of the genetic control of plasticity, and for more studies of the actual genetics of phenotypic plasticity.

\section{What do we know and what do we assume about the genetics of plasticity?}

Very few empirical studies of phenotypic plasticity have addressed its actual genetic basis. One of the classical examples is the pennant/vestigial system determining wing length and its response to temperature in Drosophila melanogaster. The reaction norms for this trait are fairly complex (figs 23 \& 24 in Schmalhausen, 1949). Yet the system is governed by only one locus with two alleles. Other apparently complex plastic phenotypes having a relatively simple genetic basis are wing dimorphism in some insects (Tauber \& Tauber, 1992; Roff \& Fairbairn, 1993), phytochrome-controlled shade-avoidance response in angiosperms (Schmitt \& Wulff, 1993), and plasticity of flowering time to vernalization in Arabidopsis thaliana (Koornneef et al., 1991).
More generally, several authors have recently pointed out the possibility that complex traits might be under simple genetic and developmental control, and that we might be placing too much emphasis on the (assumed) quantitative genetic basis of phenotypes (e.g. Gottlieb, 1984; Orr \& Coyne, 1992; Kadereit, 1994).

Several levels of criticism have been raised through the years to caution against the use of genetic correlations as tools for estimating genetic constraints in a population. These can be summarized as follows. (1) Genetic correlations are, by definition, subject to change through evolutionary time (Turelli, 1988). This is because $r_{\mathrm{G}}$ values are reflections of the actual gene frequencies of the population, which are bound to change during selection. Incidentally, this is the reason why quantitative genetic models of the evolution of plasticity assume a very weak stabilizing selection on the acrossenvironment phenotype. However, these simulations are typically carried out for thousands of generations (e.g. Via \& Lande, 1985), a span of time during which mutation pressure and drift would presumably be sufficient to alter the genetic variances and covariances. (2) Genetic correlations are affected by changes in the environment and by genotype by environment interactions, and are therefore to be considered only local statistical estimators of population properties, not useful for broad evolutionary predictions (Service \& Rose, 1985; Clark, 1987; Mazer \& Schick, 1991; Stearns et al., 1991; Ebert et al., 1993; Schlichting \& Pigliucci, 1995). (3) Genetic covariances and correlations can lead to misleading conclusions about constraints if the hierarchical structure of genetic interactions that yield the observable correlation is ignored (Houle, 1991; Gromko, 1995). The G matrix is a 'flat' representation of the correlation among characters, i.e. its entries are pairwise comparisons. If there is a hierarchical structure to the genetic architecture, i.e. if some traits are connected to others indirectly (as in metabolic flux models), then the information contained in the $\mathbf{G}$ matrix can be incomplete to say the least, and could yield misleading predictions. (4) Unlike classical genetic correlations, inter-environmental ones are actually determined by a mixture of at least two different genetic effects (if we ignore linkage): changes in gene expression catalysed by environmentally influenced regulatory genes, and changes in the allelic sensitivity of the same gene products expressed in two different environments (Schlichting \& Pigliucci, 1993). Schlichting and Pigliucci (1995) have argued that the confounding effects of these two underlying sources of variation 
can potentially generate any value for the genetic correlation between -1 and +1 .

\section{A simple heuristic model of the genetic basis of a trait and its statistical description}

The scenariograms presented here use a simple deterministic population genetic model representing the action of two alleles at one locus. The single locus controls both the average response across environments (i.e. the elevation of the reaction norm), and the pattern of plasticity (i.e. the slope of the reaction norm). The model is similar to the real example provided by the pennant/vestigial system in Drosophila melanogaster (Schmalhausen, 1949), even though it might not be representative of more complex examples of phenotypic plasticity, in which QTL analysis reveals the action of different genes in different environments (Mitchell-Olds, 1995). In the model, a population with different frequencies of the three genotypes is under stabilizing selection, in the sense that the joint across-environment optimum is within the defined range of phenotypic space. This implies that the population can 'overshoot' the optimum, as in Via (1987). Notice that the same qualitative conclusions that I will reach by examining this model are valid for any system in which a few loci elicit major effects on the phenotype, and we simply do not know at this point how frequent this case is in natural systems. A crucial point is that if it can be demonstrated that knowledge of the genetic machinery is relevant even with such a supposedly elementary system, the same argument can be applied to more complex, multilocus, situations. Finally, it is worth recalling that ANOVAS and computations of genetic correlations are carried out in empirical studies when we do not have any information about the genetic basis of plastic responses.

The model presented here assumes a panmictic population which splits at each generation into two possible environments. All gametes are then pooled before the next generation is formed (spatial heterogeneity, analogous to Via, 1987). Allelic frequencies change following two standard deterministic equations:

$$
\begin{aligned}
p_{1}^{\prime}= & ((p q(p((1-s)-(1-s h))+q((1-s h-1))) / \\
& \left.\left((1-s) p^{2}+2 p q(1-s h)+q^{2}\right)\right)+p
\end{aligned}
$$

in environment 1 , and

$$
\begin{aligned}
p_{2}^{\prime}= & ((p q(p(1-(1-s h))+q((1-s h)-(1-s))))) \\
& \left.\left(p^{2}+2 p q(1-s h)+(1-s) q^{2}\right)\right)+p
\end{aligned}
$$

in environment 2. In these equations, $p$ is the frequency of the $A$ allele, $q$ is the frequency of the $a$ allele, $s$ represents the intensity of selection, $h$ is the degree of dominance, and the primes represent the allelic frequencies of the following generation. The actual change in allelic frequencies in the whole population is the average of eqns (1) and (2), weighted using the frequency of occurrence of the two environments (similar to Levene, 1953)

$p^{\prime}=\left(1-P_{\mathrm{se}}\right) p_{1}^{\prime}+P_{\mathrm{se}} p_{2}^{\prime}$

where $P_{\mathrm{se}}$ is the probability of occurrence of the second environment. The relevant calculations can be carried out on a pocket calculator. The 'scenariograms' in Figs 1 and 2 are based on the comparison of the information that can be gathered and the inferences that can be made in each of the following three cases. (1) The first case is a two-way analysis of variance. The first two columns in each figure represent the estimates of genetic variation across treatments and of genotype by environment interactions. The first series of graphs shows the reaction norms of the three genotypes in the environment vs. phenotype $\}$ space, with the broken line representing the optimal reaction norm. (2) The second case is a 'quantitative' genetic analysis. The third column reports the estimated genetic correlation between the expression of the same trait in two environments. The second set of graphs visualizes the position of each genotype in the fphenotype in environment 1 vs. phenotype in environment 2$\}$ space. (3) The third case is given in the last two columns, which report the population genetic predictions for the evolutionary fate of the population (based on knowledge of the actual genetic machinery).

\section{First scenario: positive genetic correlations}

Let us consider first how a positive genetic correlation between the expressions of the same trait in two environments can arise in a one-locus two-allele situation. Figure 1 represents three of the many possible scenarios. The upper panels show a population with a plastic heterozygote and two nonplastic homozygotes. An analysis of variance would detect an intermediate level of genetic variation for the elevation of the reaction norm (because two of the three genotypes would have a distinct phenotypic value in either environment), and an intermediate level of $\mathrm{G}$ by $\mathrm{E}$ (because of the heterozygote). The inter-environment genetic correlation is +0.5 . If the frequency of the two environments is the same, the population genetic equations predict the evolution 


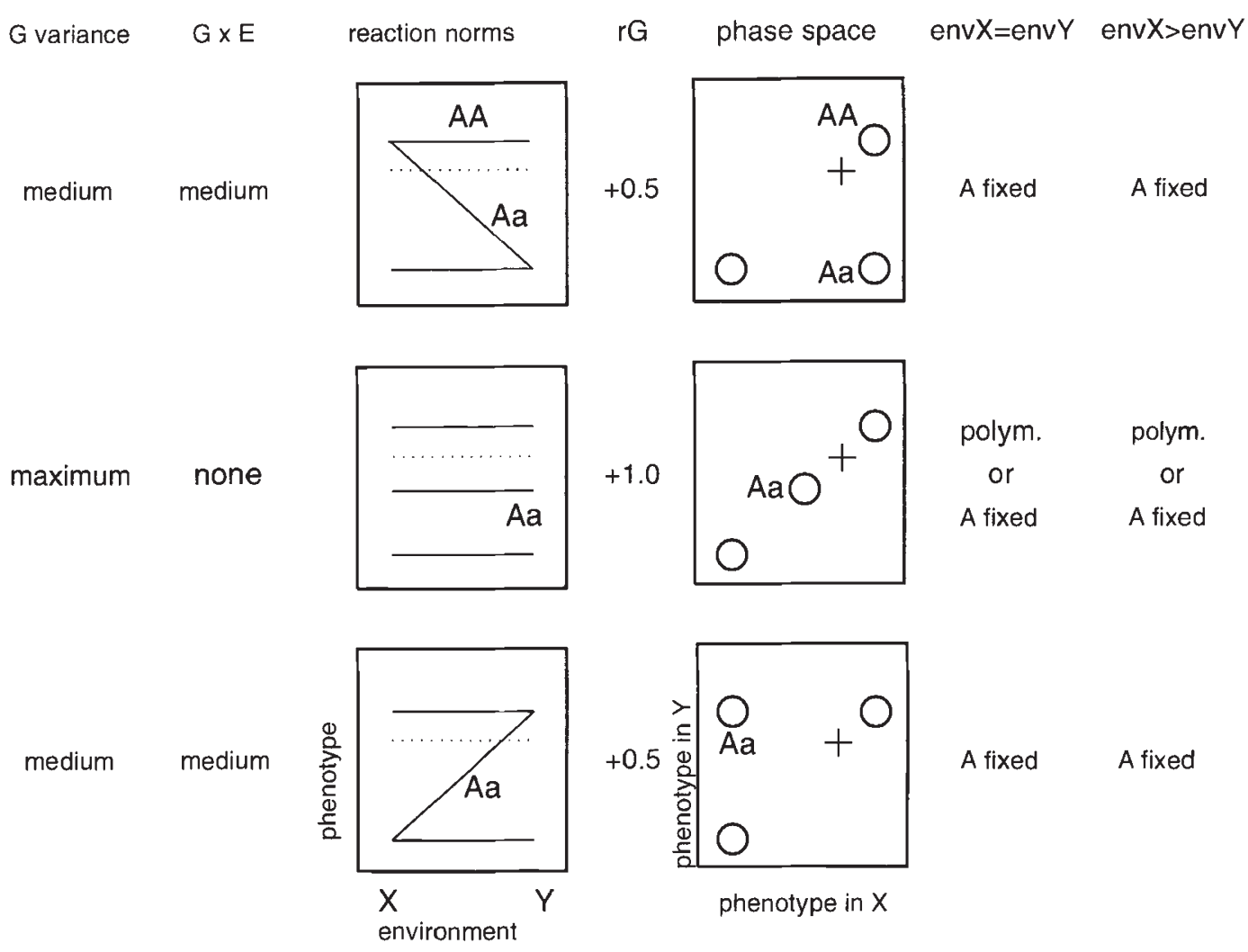

Fig. 1 Scenariogram 1: positive genetic correlation between the expressions of the same trait in two environments. The first column describes the amount of genetic variation for the elevation of the reaction norm in an analysis of variance. The second column estimates the amount of genetic variation for plasticity. The first series of graphs shows changes in the reaction norms of the three genotypes constituting the population (only the heterozygote and the $A A$ homozygote are labelled). The third column gives the genetic correlation across environments. The second set of graphs shows the same genotypes in the phase space identified by the phenotypic response in the first and in the second environment. The fourth column describes the evolutionary outcome based on the knowledge of the population genetics of the system, when the two environments occur with equal frequency. The last column describes the evolutionary outcome based on the knowledge of the population genetics of the system, when the first environment occurs more frequently than the second one.

of a stable equilibrium, with the $A$ allele becoming fixed. This is because the relative fitnesses of the three genotypes would be $W_{A A}>W_{A a}>W_{a a}$. The bottom panel illustrates the symmetrical situation, in which the heterozygote is still plastic, but is characterized by an opposite reaction norm. The population genetic equations still predict the fixation of $A$. Finally, the middle panel shows three parallel reaction norms, corresponding to the maximum possible amount of $\mathrm{G}$ variance, but no genotype by environment interaction. The genetic correlation is +1 , indi- cating a totally constrained system with no genetic variation for plasticity. Population genetics theory predicts either a polymorphism or the fixation of the $A$ allele, because the fitnesses rank as: $W_{A A}=W_{A a}$ $>W_{a a}$. Notice that in this case the final outcome would be dependent on the initial conditions: the initial frequencies of $A A$ and $A a$ would determine if a polymorphism would be maintained or not (and, in a finite population, drift would shift the outcome towards the fixation of $A$ ). The change in the genetic control of the possible phenotypes is achieved by 
altering the environment-specific relative degree of dominance of the two alleles, analogously to what has been observed in $D$. melanogaster's pennant/vestigial system.

Several observations need to be made about the situations just described. First, the ANOVA and the quantitative genetic model make apparently different predictions. When $r_{\mathrm{G}}=+1$, the system should be totally 'constrained', but the ANOVA detects a highly significant variation for the elevation in the reaction norm, implying that the system is indeed capable of responding to selective pressures. It is the plasticity, not the average response, that cannot evolve under these conditions. Secondly, a genetic correlation of +1 does in some sense constrain the system. But the efficacy of such constraint depends on where the optimum is located. In our case, the population would evolve very quickly towards the optimum, simply because it lies directly on the main diagonal, i.e. within the phenotypic space already occupied by the population. This observation was made by Via (1987), but it is usually little appreciated: the constraints implied by a genetic correlation are contextdependent, so that it does not make sense simply to say that, 'because of a significant genetic correlation, the system is constrained': indeed, because of the correlation the system could evolve faster! Finally, let us switch the labels of the $A A$ and $A a$ genotypes: whereas the outcome when $r_{\mathrm{G}}=+1$ would be identical, in the other two cases we would have the evolution of a balanced polymorphism, not the fixation of the $A$ allele, because this time the heterozygote will be much closer to the optimum than either of the other two genotypes. Hence, a change in the genetic control of the reaction norm would produce dramatically different evolutionary outcomes, even though neither the analysis of variance nor the quantitative genetic model would detect any alteration in the statistical distribution of the population (contrary to Via, 1993).

What happens if the two environments occur with different frequencies? The outcomes listed in the rightmost column of Fig. 1 concern the case in which the frequency of the first environment is greater than that of the second environment. Obviously, neither the ANOVA nor the genetic correlation are affected by the change in environmental frequency. The evolutionary outcomes of the three situations also mirror the ones already discussed. Thus, when $r_{\mathrm{G}}$ is positive, the frequency of occurrence of the environments does not affect the evolutionary equilibrium. As we shall see, this is no longer true when the genetic correlation becomes negative.

(C) The Genetical Society of Great Britain, Heredity, 77, 453-460.

\section{Second scenario: negative genetic correlations}

A simple population genetic situation yielding negative genetic correlations between environments is illustrated in Fig. 2. The central panel shows a resulting genetic correlation of -1 : the three genotypes are aligned along the main alternate diagonal in phenotypic phase space, so that a genotype with a high phenotypic expression in the first environment is characterized by a low phenotypic expression in the second one, or vice versa. Throughout the figure, the heterozygote is always not plastic, but the elevation of its reaction norm changes in the three panels. When the genetic correlation is -1 , the analysis of variance detects no genetic variation for the elevation of the reaction norm, but the highest possible value of $G$ by $E$. Here, the simplest interpretations of the two models are again in apparent contrast: a value of -1 for $r_{\mathrm{G}}$ would be taken as indication of a totally constrained system. Correspondingly, the ANOVA would suggest no possibility of evolution of the across-environment mean. However, the analysis of variance would also imply a very strong genetic variation for plasticity, meaning that the slope of the reaction norm should respond to selection. Once again, constraints are contextspecific: if the optimum lies outside the alternate diagonal, then the population will resist evolutionary change, or change in a maladaptive fashion (Via, 1987). But if the optimum is within the region of phenotypic space defined by the alternate diagonal, evolution would be both adaptive and very fast, this time because of a change in plasticity. Given the assumed genetic constitution of the population, the population genetic model predicts the evolution of a stable polymorphism, both in the case of equiprobable environments and when the frequency of the first environment is higher (because $W_{A a}>W_{A A}=W_{a a}$ ) (Fig. 2, rightmost column).

The top and lower panels of Fig. 2 illustrate two cases yielding a genetic correlation of -0.5 . In the top panel, this is achieved when the heterozygote has the highest phenotypic response in both environments, in the bottom panel when it has the lowest phenotypic response across environments. Again, two very different genetic mechanisms can appear to yield exactly the same population-level situation when analysed statistically. The analysis of variance would detect an intermediate amount of $G$ variance and of $\mathrm{G}$ by $\mathrm{E}$ in both cases. Therefore, both the ANOVA and the quantitative genetic approach would predict a mildly constrained system. However, population genetics shows us that the evolutionary trajec- 


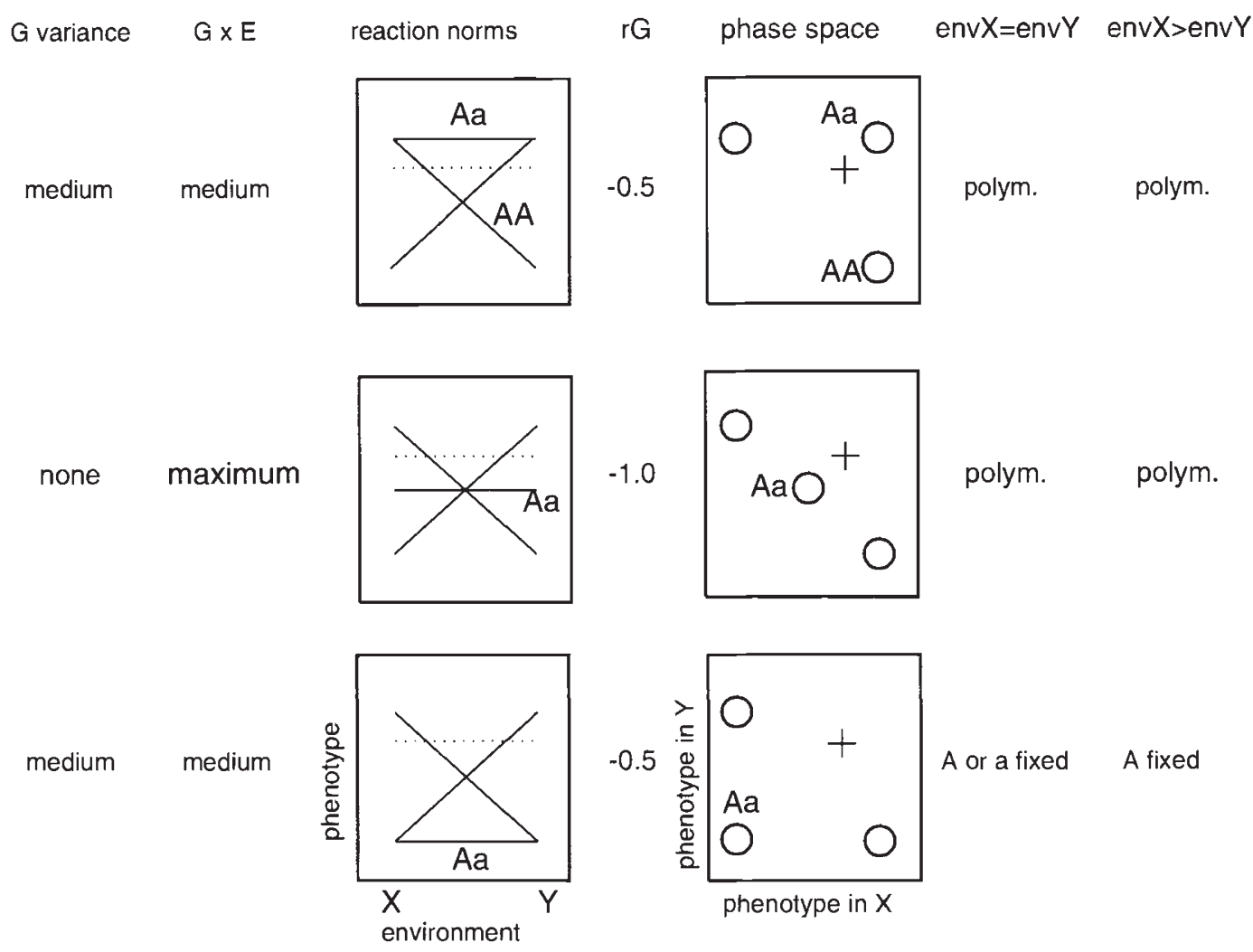

Fig. 2 Scenariogram 2: negative genetic correlation between the expressions of the same trait in two environments (legend as in Fig. 1).

tory would depend on how exactly the genetic correlation is achieved, as well as on what the frequencies of the two environments are. In the case of equally frequent environments, we will have a polymorphic equilibrium analogous to the one achieved before, if the heterozygote is closest to the optimum. When the heterozygote is far from the optimum, however, the system will evolve towards the fixation of either $A$ or $a$, depending on the initial conditions (because $W_{A A}=W_{a a}>W_{A a}$ ). Furthermore, if the two environmental frequencies are unequal, the same polymorphism would arise when the heterozygote is closest to the optimum, but the alternate scenario could bring only the fixation of $A$ (because now $W_{A A}>W_{A a}>W_{a a}$ ). We can again perform the heuristic exercise of exchanging the labels of $A A$ and $A a$. When the environmental frequencies are unequal and we consider the two cases characterized by $r_{\mathrm{G}}=-0.5$, the system will now yield one fixed equilibrium (instead of a polymorphism) in the top panel, and a polymorphism (instead of a fixed equilibrium) in the lower panel of Fig. 2. Therefore, when the genetic correlation between environments is negative, the frequency of the two environments does affect the evolutionary outcome, unlike the scenario when $r_{\mathrm{G}}$ is positive.

\section{Conclusions}

The whole rationale of evolutionary quantitative genetics is that we need to resort to a statistical (as opposed to a mechanistic) description of evolution because population genetic-type approaches become too complex for anything more than two loci, and because we are generally ignorant of the real genetic 
basis of a given trait. But the power of quantitative genetic analysis is gained at the cost of an extreme simplification of the biology of the system. We are then faced by fundamental empirical questions: how complex are the genetic bases of actual phenotypes? What kind of complexity should we consider, the often assumed one emerging from many loci with simple effects, or the sometimes documented one characterizing few loci with interactions? At the same time, we should seriously discuss what the role of models in biology is or can be. In the field of phenotypic plasticity the number of theoretical papers almost surpasses that of experimental efforts. Although this speaks for the vitality and conceptual interest of the discipline, it might also yield a situation in which we will have a plethora of untestable models claiming to have 'demonstrated' something. It is my conviction that models, like any theoretical statement, are valuable only when they can be falsified. If their role becomes that of constraining the conclusions of empirical studies, they amount to deleterious intellectual exercises. It might ultimately be impossible to come up with models that are at the same time general enough to be of broad intellectual appeal while retaining predictive power, given the complexity of biological phenomena (Kauffman, 1993). We might have to learn how to live with this conclusion.

\section{Acknowledgements}

I wish to thank Guido Barbujani, Kent Holsinger, Dave Rand, and Carl Schlichting for critical comments on previous versions of this manuscript. This work was partially supported by NSF dissertation improvement grant DEB-9122762 and by a Sigma-Xi Research-in-Aid grant.

\section{References}

ABRAMS, P. A., HARADA, Y. AND MATSUDA, H. 1993. On the relationship between quantitative genetic and ESS models. Evolution, 47, 982-985.

ANDERSSON, S. AND SHAW, R. G. 1994. Phenotypic plasticity in Crepis tectorum (Asteraceae): genetic correlations across light regimens. Heredity, 72, 113-125.

BRADSHAW, A. D. 1965. Evolutionary significance of phenotypic plasticity in plants. Adv. Genet., 13, 115-155.

CHARLESWORTH, B. 1990. Optimization models, quantitative genetics, and mutation. Evolution, 44, 520-538.

CLARK, A. G. 1987. Senescence and the genetic-correlation hang-up. Am. Nat., 129, 932-940.

CONNER, J. AND VIA, S. 1993. Patterns of phenotypic and genetic correlations among morphological and lifehistory traits in wild radish, Raphanus raphanistrum.
Evolution, 47, 704-711.

DE JONG, G. 1990. Quantitative genetics of reaction norms. J. Evol. Biol., 3, 447-468.

DE JONG, G. 1995. Phenotypic plasticity as a product of selection in a variable environment. Am. Nat., 145, 493-512.

EBERT, D., YAMPOLSKY, L. AND VAN NOORDWIJ, A. J. 1993. Genetics of life history in Daphnia magna. II. Phenotypic plasticity. Heredity, 70, 344-352.

FALCONER, D. S. 1952. The problem of environment and selection. Am. Nat., 86, 293-298.

FALCONER, D. S. 1990. Selection in different environments: effects on environmental sensitivity (reaction norm) and on mean performance. Genet. Res., 56, 57-70.

GAVRILETS, S. AND SCHEINER, s. M. 1993. The genetics of phenotypic plasticity. V. Evolution of reaction norm shape. J. Evol. Biol., 6, 31-48.

GEBHARDT, M. D. AND STEARNS, S. C. 1993. Phenotypic plasticity for life history traits in Drosophila melanogaster. I. Effect on phenotypic and environmental correlations. J. Evol. Biol., 6, 1-16.

GILlespie, J. H. AND TURELli, M. 1989. Genotype-environment interactions and the maintenance of polygenic variation. Genetics, 121, 129-138.

GOMULKIEWICZ, R. AND KIRKPATRICK., M. 1992. Quantitative genetics and the evolution of reaction norms. Evolution, 46, 390-411.

GOTtLiEB, L. D. 1984. Genetics and morphological evolution in plants. Am. Nat., 123, 681-709.

GROMKO, M. H. 1995. Unpredictability of correlated response to selection: pleiotropy and sampling interact. Evolution, 49, 685-693.

HOULE, D. 1991. Genetic covariance of fitness correlates: what genetic correlations are made of and why it matters. Evolution, 45, 630-648.

KADEREIT, J. W. 1994. Molecules and morphology, phylogenetics and genetics. Bot. Acta, 107, 369-373.

Kauffman, s. A. 1993. The Origins of Order. Oxford University Press, Oxford.

KOORNNEEF, M., HANHART, C. J. AND VAN DER VEEN, J. H. 1991. A genetic and physiological analysis of late flowering mutants in Arabidopsis thaliana. Mol. Gen. Genet., 229, 57-66.

LEVENE, H. 1953. Genetic equilibrium when more than one ecological niche is available. Am. Nat., 77, 331-333.

LEWONTIN, R. C. 1974. The analysis of variance and the analysis of causes. Am. J. Hum. Genet., 26, 400-411.

MAZER, S. J. AND SCHICK, C. T. 1991. Constancy of population parameters for life history and floral traits in Raphanus sativus L. I. Norms of reaction and the nature of genotype by environment interactions. Heredity, 67, $143-156$.

MITCHELL-OLDS, T. 1995. The molecular basis of quantitative genetic variation in natural populations. Trends Ecol. Evol., 10, 324-327.

ORR, H. A. AND COYNE, J. A. 1992. The genetics of adaptation: a reassessment. Am. Nat., 140, 725-742.

PIGLIUCCI, M. 1992. Modelling phenotypic plasticity. I. Linear and higher order effects of dominance, drift, 
environmental frequency and selection on a one-locus, two-allele model. J. Genet., 71, 135-150.

ROFF, D. A. AND FAIRBAIRN, D. J. 1993. The evolution of alternate morphologies: fitness and wing morphology in male sand crickets. Evolution, 47, 1572-1584.

SCHEINER, S. M. 1993. Genetics and evolution of phenotypic plasticity. Ann. Rev. Ecol. Syst., 24, 35-68.

schlichting, C. D. 1986. The evolution of phenotypic plasticity in plants. Ann. Rev. Ecol. Syst., 17, 667-693.

sCHLichting, C. D. AND PIGLIUCCl, M. 1993. Control of phenotypic plasticity via regulatory genes. Am. Nat., 142, 366-370.

SCHLichting, C. D. AND PIGLiUCCI, M. 1995. Gene regulation, quantitative genetics, and the evolution of reaction norms. Evol. Ecol., 9, 154-168.

SChmalhausen, I. I. 1949. Factors of Evolution. Blakiston, Philadelphia.

SCHMITT, J. AND WULFF, R. 1993. Light spectral quality, phytochrome and plant competition. Trends Ecol. Evol., 8, 47-50.

SERVICE, P. M. AND ROSE, M. R. 1985. Genetic covariation among life-history components: the effect of novel environments. Evolution, 39, 943-945.

STEARNS, S., JONG, G. D. AND NEWMAN, B. 1991. The effects of phenotypic plasticity on genetic correlations. Trends Ecol. Evol., 6, 122-126.

SUlTAN, s. E. 1987. Evolutionary implications of phenotypic plasticity in plants. Evol. Biol., 21, 127-178.

TAuber, C. A. And TAuber, M. J. 1992. Phenotypic plasti- city in Chrysoperla: genetic variation in the sensory mechanism and in correlated reproductive traits. Evolution, 46, 1754-1773.

TURELli, M. 1988. Phenotypic evolution, constant covariances, and the maintenance of additive variance. Evolution, 42, 1342-1347.

VAN TIENDEREN, P. H. AND KOELEWIJn, H. P. 1994. Selection on reaction norms, genetic correlations and constraints. Genet. Res., 64, 115-125.

VIA, s. 1987. Genetic constraints on the evolution of phenotypic plasticity. In: Loeschcke, V. (ed.) Genetic Constraints on Adaptive Evolution, pp. 47-71. SpringerVerlag, Berlin.

V1A, s. 1993. Adaptive phenotypic plasticity: target or by-product of selection in a variable environment? Am. Nat., 142, 352-365.

VIA, s. AND LANDE, R. 1985. Genotype-environment interaction and the evolution of phenotypic plasticity. Evolution, 39, 505-522.

West-eberhard, M. J. 1989. Phenotypic plasticity and the origins of diversity. Ann. Rev. Ecol. Syst., 20, 249-278.

WINDIG, J. J. 1994. Genetic correlations and reaction norms in wing pattern of the tropical butterfly Bicyclus anynana. Heredity, 73, 459-470.

WOLTERECK, R. 1909. Weitere experimentelle Untersuchungen über Artveränderung, speziell über Wesen quantitativer Artunterschiede bei Daphniden. Verh. D. Tsch. Zool. Ges., 1909, 110-172.

YAMADA, Y. 1962. Genotype $\times$ environment interaction and genetic correlation of the same trait under different environments. Jap. J. Genet., 37, 498-509. 